\title{
Weight loss in obese patients with asthma improved lung function and health status
}

Stenius-Aarniala B, Poussa T, Kvarnström J, et al.Immediate and long term effects of weight reduction in obese people with asthma: randomised controlled study. BMJ 2000 Mar 25;320:827-32.

\section{QUESTION: In obese patients with asthma, does weight loss improve lung function and health status and alleviate symptoms?}

Design

Randomised (allocation concealed*), unblinded,* controlled trial with 1 year follow up.

\section{Setting}

Outpatient clinic in Helsinki, Finland.

\section{Patients}

38 patients who were $18-60$ years of age (mean age $49 \mathrm{y}, 76 \%$ women), had a body mass index of 30 to 42 , had asthma with a spontaneous diurnal variation or a bronchodilator response of $\geqslant 15 \%$, and were nonsmokers. Exclusion criteria were pregnancy; history of bulimia or anorexia; other severe disease, including heart, thyroid, liver, or gallbladder disorders; insulin or systemic steroid treatment; or history of food allergy or intolerance to any element of the intervention diet. Follow up was complete.

\section{Intervention}

Patients were allocated to a weight loss group $(n=19)$ or a control group $(n=19)$. The weight reduction programme included 12 group therapy sessions in 14 weeks, including an 8 week dieting period in which patients took a very low energy dietary preparation (Nutrilett, Nycomed Pharma, Oslo, Norway) that provided $1760 \mathrm{~kJ}$ of energy per day. Control group patients had non-specific group sessions that included education about asthma and allergy and were held at the same times as those of the weight loss group.

\section{Main outcome measures}

Change in body weight, peak expiratory flow (PEF), $\mathrm{FEV}_{1}$, forced vital capacity (FVC), asthma symptoms, and health status.

For correspondence: Dr B Stenius-Aarniala Department of

Medicine, University Central Hospital, PL 340 Helsinki, FIN-00029 HYKS,

Finland. respectively, in the control group. The groups did not differ for change in PEF at any point during follow up $(\mathrm{p} \geqslant 0.06)$, but patients in the weight loss group had greater increases than control group patients for $\mathrm{FEV}_{1}$ and FVC at all follow up time points $(p \leqslant 0.02)$ ( 1 y results are in the table). At programme's end, the weight loss group had greater reductions in dyspnea and use of rescue medication, but the difference did not reach statistical significance by 1 year. Patients in the weight loss group had greater improvement in overall health status than control group patients $(\mathrm{p}=0.02)$.

\section{Conclusion}

In obese patients with asthma, weight loss improved lung function and health status.

*See glossary.

\section{COMMENTARY}

The role of weight loss in improving the quality of life of people with asthma has not been extensively studied. Thus, the study by Stenius-Aarniala $e t$ al of 38 patients from a hospital outpatient setting provides useful insight into the value of intensive weight reduction. However, several issues that affect the external validity of the study need to be discussed, and the hospital outpatient setting of the study raises concerns about the generalisability of the results to primary care.

The results suggest that overweight people with asthma may benefit from the combination of weight reduction and optimal pharmacological management. It is well known, however, that the problem with intense interventions in patients with asthma is sustainability, which raises doubts about the long term effects of this type of intervention. Statistically significant differences existed between the control and intervention groups, but their clinical relevance remains unclear. The changes in PEF, $\mathrm{FEV}_{1}$, and $\mathrm{FVC}$ in the intervention group were small. If the health status measures are examined in detail, the only items that increased to a statistically significant extent were overall score and symptom control, whereas activity and social and psychological effects did not. Patient time and the cost of the commitment to such an intensive programme were not considered. Further studies are needed to explore these concerns in detail.

Justin Beilby, MBBS, MD, MPH University of Adelaide Adelaide, South Australia, Australia 